\title{
Papel do enfermeiro da atenção básica de saúde na abordagem ao dependente de drogas em João Pessoa, PB, Brasil
}

\author{
Nurse's role on basic health care regarding to the approach to drug addicted in João Pessoa, PB, Brazil
}

Rol del enfermero de la atención básica de salud sobre el abordaje a los usuarios de drogas en João Pessoa, PB, Brasil

\section{Karelline Izaltemberg Vasconcelos Rosenstock', Maria José das Neves'}

'Universidade Federal da Paraíba. Centro de Ciências da Saúde. Departamento de Enfermagem. João Pessoa, PB

Submissão: 26/04/2009

Aprovação: 09/07/2010

\section{RESUMO}

Estudo de abordagem eualitativa realizado com enfermeiras da Estratégia Saúde da Família em João Pessoa, PB, brasil. O objetivo foi investigar o papel destes enfermeiros na abordagem aos dependentes de drogas, analisando estratégias de cuidados básicos de enfermagem aplicados a estes usuários. A seleção para participar do estudo seguiu o critério de aceitação. Os dados foram coletados através de entrevista semi-estruturada, priorizando a fala dos participantes. Constatou-se a carência na formação profissional dos enfermeiros em relação à dependência de drogas, restringindo suas ações ao encaminhamento dos usuários a serviços mais especializados em saúde mental. Deve-se redefinir a atenção, colocando um novo paradigma de saúde como direito e construção social comunitária, a partir de uma visão globalizante dos processos de interação social.

Descritores: Enfermagem; Transtornos relacionados ao uso de substâncias; Programa Saúde da Família.

\section{ABSTRACT}

Qualitative study conducted with nurses from the Family Health Strategy in João Pessoa, PB, Brasil. The purpose was to investigate the nurses' role in the approach to the drug addict, analyzing the strategies of basic nursing care applied to them. The selection for the study followed the criterion for acceptance. The data were collected through a semi-structured interview, giving priority to participant's talks. One noticed the lack of professional training of the nurses in relation to the drug addiction, by restricting their actions to taking these users to more specialized services in mental health. It must redefine the attention, putting a new paradigm of health as a right and as a social community construction, from a global view of the processes of social interaction.

Key-words: Nursing; Substance-related disorders; Family Health Program.

\section{RESUMEN}

Estudio de abordaje cualitativo, realizado con personal de enfermería en la Estrategia de Salud de la Familia en João Pessoa/PB. Lo objetivo fue investigar el rol de los enfermeros en el enfoque para dependientes de las drogas, el examen de las estrategias básicas para la atención de enfermería aplicado a estos usuarios. La selección para el estudio siguió el criterio de aceptación. Los datos fueron recolectados a través de entrevistas semi-estructuradas, centrándose el discurso de los participantes. Es la falta de formación de los enfermeras sobre dependencia a drogas, limitando sus acciones de la ruta de los usuarios de los servicios más especializados en salud mental. Usted debe reiniciar el centro, poniendo un nuevo paradigma de la salud como el derecho comunitario y de construcción social, desde una visión global de los procesos de interacción social.

Descriptors: Enfermería; Trastornos relacionados con sustancias; Programa Salud de la Familia.

AUTOR CORRESPONDENTE Karelline Izaltemberg Vasconcelos Rosenstock. Universidade Federal da Paraíba. Centro de Ciências da Saúde. Cidade Universitária. CEP 58059-900. João Pessoa, PB. E-mail: mrosenstock@ig.com.br 


\section{INTRODUÇÃO}

A diversidade de problemas e de pessoas envolvidas com as drogas permite dizer que o abuso de substâncias psicoativas é um problema de saúde pública da maior importância. Segundo a Organização Mundial de Saúde, em 2004, cerca de 10\% das populações dos centros urbanos de todo o mundo, consomem abusivamente substâncias psicoativas independentemente de idade, sexo, nível de instrução e poder aQuisitivo; a dependência Química é determinada por uma série de motivos, todos com papel importante, como: fatores biológicos, genéticos, psicossociais, ambientais e culturais ${ }^{(1)}$. A dependência de drogas é um estado mental e, muitas vezes, físico, Que resulta da interação entre um organismo vivo e uma droga psicoativa, e sempre inclui uma compulsão de usar a droga para experimentar seu efeito psíQuico ou evitar o desconforto provocado pela sua ausência ${ }^{(2)}$.

No Brasil, esse problema é avaliado de acordo com a freeüência de internações em instituições psiQuiátricas e pesQuisas realizadas no meio estudantil. No Que se refere a internações o uso do álcool foi o principal causador de internações por transtorno mental no país entre 1988 e 1999, respondendo por $90 \%$ dessas, segundo o II Levantamento Domiciliar sobre o uso de drogas psicotrópicas no Brasil, realizada em 2005, pelo Centro Brasileiro de Informações sobre Drogas Psicotrópicas ${ }^{(3)}$. A pesquisa também revelou, no mesmo período, um aumento de $4,7 \%$ para $15,5 \%$ na proporção das internações provocadas pela dependência de outras drogas. No período, as internações por uso de cocaína passaram de $0,8 \%$ para $4,6 \%$.

De acordo com o Ministério da Saúde, as Queixas psíquicas são a segunda causa mais frequente de procura por atendimento na Atenção Básica, as experiências de atendimento e acolhimento das demandas de saúde mental é algo constatado por 56\% das equipes da Saúde da Família; os dados epidemiológicos apontam Que de 6 a $8 \%$ da população necessite de algum cuidado decorrente do uso prejudicial do álcool ou outras drogas ${ }^{(4)}$.

O Programa Saúde da Família - PSF, institucionalizado em 1994 pelo Ministério da Saúde do Brasil, foi criado com o intuito de reverter o modelo assistencial vigente, Que era o hegemônico, centrado em ações de cura e no ato médico ${ }^{(5)}$. A partir de 2006, o Ministério da Saúde passou a denominar o PSF como Estratégia de Saúde da Família (ESF), por meio da Portaria ${ }^{\circ}$ 648/06 ${ }^{(6)}$. A alteração deu-se, a fim de fortalecer a idéia da Saúde da Família como o eixo estruturante da atenção básica, e não apenas como sendo mais um programa entre outros propostos pelo Ministério da Saúde. A universalização da atenção à saúde, garantida pelo SUS e, consequentemente, pela ESF, vem proporcionado a milhões de brasileiros uma acessibilidade e acesso a ações e serviços saúde, e é este modelo de atenção à saúde Que vem permitindo a maior inclusão social, política e econômica a uma política sanitária ${ }^{(5)}$.

Dentre os profissionais de saúde, os enfermeiros são os Que mantêm contato maior com os usuários dos serviços de saúde, tendo grande potencial para reconhecer os problemas relacionados ao uso de drogas e desenvolver ações assistenciais. A principal vantagem da Atenção Básica é a possibilidade de estender a cobertura em saúde mental a um maior número de usuários e diminuir os encaminhamentos de pacientes menos graves para a atenção especializada ${ }^{(7)}$.
Em um primeiro contato, o enfermeiro é capaz de conhecer a história atual do uso de substâncias psicoativas, padrão de consumo da substância e, ciente dos problemas relacionados ao uso, pode realizar o acolhimento e breve sensibilização, pelo confronto dos problemas relatados pelo paciente e sua associação com o uso da substância. Há poucas pesquisas abordando a formação de profissionais de saúde, dentre eles os enfermeiros, nesta área, a despeito da demanda de pacientes e da gravidade dos problemas, os Quais solicitam habilidade específica e encaminhamentos multidisciplinares $^{(8)}$

O presente estudo tem como objetivo investigar o papel do enfermeiro da Atenção Básica de Saúde na abordagem ao dependente de drogas, identificando as atividades desenvolvidas por estes profissionais no atendimento destes usuários, bem como as estratégias de cuidados básicos de enfermagem aplicados a eles. Este estudo se mostra pertinente devido ao problema trazido pela dependência de drogas, sendo este tão nocivo à sociedade Quanto ao indivíduo, e o papel do enfermeiro não está ligado somente ao tratamento de usuários e sua respectiva doença, mas também possui um caráter de educação preventiva, informação e re-introdução social.

Portanto, a importância da intervenção da enfermagem, enQuanto estratégia baseada na recuperação e estabilização do dependente é de suma relevância, uma vez Que esse profissional terá mais oportunidades de acesso ao dependente, principalmente em momentos de maior fragilidade, isto é, Quando ele procura ajuda necessitando ser acolhido e tratado adequadamente.

A análise do material convergiu para a construção das seguintes categorias empíricas:

- O enfermeiro e os cuidados de saúde no âmbito da Atenção Básica de Saúde;

- O cuidado de enfermagem na abordagem ao usuário de drogas.

\section{METODOLOGIA}

O estudo foi de caráter exploratório e de abordagem Qualitativa, priorizando a fala dos participantes. A aplicação da metodologia Qualitativa no campo da saúde torna-se importante, sobretudo por Que "o objeto saúde oferece um nível possível de ser Quantificado mas ultrapassa Quando se trata de compreender dimensões profundas e significativas Que não conseguem ser aprisionadas em variáveis"(9).

A peseuisa foi realizada na cidade de João Pessoa, a capital e o município mais populoso do estado brasileiro da Paraíba, sua população perfaz um total de 674.762 habitantes $^{(10)}$. A rede de serviços de saúde do município de João Pessoa está distribuída territorialmente em cinco Distritos Sanitários que recortam toda a extensão territorial da cidade. Os locais escolhidos para a realização desta pesquisa foram as Unidades de Saúde da Família do Timbó I, Timbó II e Bancários, todas inseridas no Distrito Sanitário III Que conta com trinta enfermeiros Que atuam nas USF's. A amostra foi constituída por três enfermeiras lotadas nestas Unidades, e a seleção para participar do estudo seguiu o critério de aceitação dos participantes.

Antes de iniciarmos a coleta em campo, seguindo as normatizações da Comissão Nacional de Ética em Pesquisas CONEP, presentes na resolução do CNS 196/96 e Capítulo IV da 
Resolução 25 1/97, o projeto de pesQuisa passou pelo Comitê de Ética em Pesquisa do Centro de Ciências da Saúde da Universidade Federal da Paraíba, tendo sido aprovado sem ressalvas segundo o protocolo $\mathrm{n}^{\circ} 0215-08$.

Os dados foram coletados através de entrevista semi-estruturada e na ocasião foi entregue o Termo de Consentimento Livre e Esclarecido aos participantes do estudo Que os assinaram. O roteiro das entrevistas foi previamente elaborado e continha as seguintes perguntas:

a) Qual é o papel do enfermeiro na Atenção Básica?

b) Qual é o papel do enfermeiro da Atenção Básica na abordagem ao indivíduo usuário de drogas?

c) Quais estratégias de cuidado de enfermagem são aplicadas no atendimento destes indivíduos?

d) Relate uma experiência recente em Que realizou atendimento de enfermagem a um indivíduo usuário de drogas.

As entrevistas foram gravadas e logo em seguida foram transcritas, os sujeitos foram nomeados de El, E2 e E3, e as entrevistas tiveram duração de 8'38', 27'17' e 11'40", respectivamente. Em seguida foram agrupadas as falas Que se assemelhavam com relação à idéia central e suas expressões-chave correspondentes. Na análise e interpretação dos dados coletados foi utilizada a técnica da análise de discurso proposta por Fiorin. O discurso "é a combinação de elementos lingüísticos (frases ou conjuntos constituídos de muitas frases) usados pelos falantes com o propósito de exprimir seus pensamentos"(1I).

\section{RESULTADOS E DISCUSSÃO}

\section{A enfermagem e os cuidados de saúde no âmbito da Atenção Básica de Saúde}

A análise do material empírico revelou as concepções dos enfermeiros a cerca do papel da enfermagem na Atenção Básica de Saúde, representado na categoria cuidados de saúde, com o significado de cuidado integral, um dos fundamentos preconizados pelo SUS; observamos a compreensão das ações desses profissionais associada a "prevenção, promoção da saúde, a escuta, o apoio", conforme os depoimentos:

O meu papel aQui no PSF é o atendimento em todo ciclo de vida do indivíduo, a gente tem um trabalho desde a criança Que está na maternidade, né, no ventre da mãe [...] a Questão do planejamento familiar, a Questão do drogadicto, a saúde mental, a prevenção e promoção da saúde, né, e tudo isso (El).

[...] nós enfermeiras somos cuidadoras, não somos 'curadoras', né, é o acolhimento, a prevenção, a escuta, o apoio, o material, ou seja, cuidar do indivíduo de todas as formas. (E2)

O enfermeiro na atenção básica hoje tem um papel imprescindível, [...] as atividades que a gente faz no dia-dia dentro da unidade de saúde no sentido de prevenção, promoção e até mesmo a Questão da reabilitação, são muitas atribuições. (E3)

O discurso dos três sujeitos confirma o conhecimento sobre a ação do enfermeiro no campo de atividades da assistência integral à saúde, um dos princípios filosóficos do SUS, sendo essas atividades desenvolvidas no sentido da promoção e proteção da saúde, prevenção de agravos, diagnóstico, tratamento, reabilitação e manutenção da saúde aos indivíduos e famílias nas Unidades de Saúde da Família e, Quando indicado ou necessário, no domicílio ou nos demais espaços comunitários; em todas as fases do desenvolvimento humano ${ }^{(12)}$.

Ao analisar os depoimentos dos enfermeiros no âmbito da Saúde Coletiva, verificou-se o enfoque ao indivíduo "dentro do contexto Que ele vive", conforme os depoimentos:

[...] a atenção do indivíduo no domicílio, e a atenção deste indivíduo no contexto da família e no contexto sócio-econômico, e na comunidade, [...] você tem uma clientela definida e, ao longo do tempo, vai conhecendo o nome, pessoas, e os contextos familiares, e todas as problemáticas Que existem. (E I)

Quando o enfermeiro consegue ter um vínculo bom com a comunidade ele consegue fazer aQuelas atribuições Que são determinadas ao enfermeiro e muito mais, para realizar ações Que realmente interfiram na vida do indivíduo, devemos olhar pra ele como um todo, inserido na comunidade e na sociedade. (E2)

A primeira coisa importante na atuação do enfermeiro na Atenção Básica é a Questão do vínculo com a comunidade, [...] você conhecer a comunidade, aquele usuário dentro do contexto que ele vive, de tudo, e não tentar resolver aquilo que ele está sentindo no momento, às vezes uma dor, sem saber ao certo o Que está acontecendo com ele lá na sua moradia, na comunidade. (E3)

A atribuição dos profissionais de saúde como agentes de mudança no contexto de atenção à família passa a ser de facilitadores no processo da educação em saúde. Os depoimentos em análise manifestam, portanto, este modelo de assistência proposto pelo SUS, Que são as necessidades sociais de saúde, as Quais o trabalho deve estar coerentemente articulado para atendê-las.

A dependência Química vem se impondo como um problema de saúde pública e como tal, reQuer um modelo de atenção incluindo a promoção da saúde, o enfoQue na prevenção do uso e abuso visando produzir as transformações sociais Que propiciem uma melhor Qualidade de vida da sociedade como um todo.

Os profissionais da Atenção Básica, convivendo com a comunidade em Que atuam, podem desencadear mudanças significativas na sua área de abrangência, se observarem o cotidiano dessas pessoas com base nas teorias e conceitos do SUS. Sob esse aspecto, as atribuições fundamentais desses profissionais da Atenção Básica são o planejamento de ações de saúde, promoção e vigilância; trabalho interdisciplinar em equipe e abordagem integral da família ${ }^{(12)}$.

\section{O cuidado de enfermagem na abordagem ao usuário de drogas}

A partir da análise do material empírico, foi possível identificar, na categoria de estratégias do cuidado de enfermagem ao usuário de drogas, a deficiência na experiência prática e teórica do enfermeiro Quanto aos assuntos referentes ao uso e a dependência de substâncias psicoativas, bem como sobre os usuários, tornando 
muito limitada a intervenção efetiva deste profissional, isso pode ser visto nos depoimentos a seguir:

A gente não tem nada Que nos oriente teoricamente direcionado ao drogadicto, eu acho Que essas ações são feitas de acordo com o conhecimento nosso no dia-dia [...] nós não temos alcoolistas, os fumantes Que a gente tem são pessoas Que são hipertensas e diabéticas, e aí a gente tem indicação para aquele programa anti-tabagista, Que acontece no CAIS, [...] os malefícios eles conhecem todos. (E I)

Com relação ao indivíduo usuário de drogas, eu me sinto totalmente perdida assim como eles, [...] eu tenho muito medo de lidar com o uso de drogas dentro de uma comunidade violenta, então você tem medo de mexer naquilo, é como se fosse um vespeiro, você tem medo de colocar a mão ali, o problema é se envolver e depois virem outras coisas piores, como os traficantes, não é só o usuário, é toda a rede do tráfico por trás disso, (E2)

Eu ainda sinto muita dificuldade com relação a isso, são duas coisas que eu sinto uma dificuldade, mas eu acho que a gente tem que trabalhar todo dia Que é: a Questão do usuário de drogas e a Questão dos pacientes com problemas mentais, eu não posso falar por todos, mas eu, mesmo hoje com o curso de terapia comunitária, não me sinto preparada para trabalhar com estes usuários. (E3)

A partir destas colocações, podemos observar a falta de preparo destes profissionais, a enfermagem está vinculada ao processo de transformação sócio-econômica e cultural do sistema. Observamos a carência em relação aos treinamentos (educação continuada) ou mesmo o ensino formal dessa temática das drogas, a Qual tem sido pouco explorada nos currículos de graduação em enfermagem, sendo considerados indispensáveis na formação dos enfermeiros com vistas à oferta de uma assistência Qualificada a essa população.

Para tanto, é fundamental a inserção do enfermeiro na equipe de saúde colaborando no enfrentamento do problema, mas há necessidade de uma ampla estrutura de conhecimento sobre promoção e prevenção para a saúde de toda a sociedade e as medidas de prevenção do uso e abuso de todas as drogas lícitas e ilícitas ${ }^{(13)}$. O desconhecimento sobre as especificidades Que envolvem o uso de drogas e da dependência Química dos técnicos de saúde é produto de uma formação acadêmica e profissional Que super estima e super valoriza as normas e regras em detrimento das atitudes sociais e valores éticos mais adequados a culturas determinadas ${ }^{(14)}$.

Ao atuar com usuários de drogas o enfermeiro deve pesquisar, provar e testar modos de cuidar Que sejam resolutivos, sem perder a característica humana no processo ${ }^{(15)}$. A Terapia Comunitária, por exemplo, é um espaço onde se procura partilhar experiências de vida e sabedorias de forma horizontal e circular, cada um tornase terapeuta de si mesmo, a partir da escuta das histórias de vida Que ali são relatadas ${ }^{(16)}$. Neste espaço o enfermeiro é capaz de reconhecer os problemas relacionados ao uso de drogas, bem como realizar o acolhimento e breve sensibilização, pelo confronto dos problemas relatados pelo usuário e sua associação com o uso de drogas.
Nesse sentido, há de se deixar de lado o conceito de Que a dependência é um problema do usuário, abordando-o como uma Questão Que afeta a todos: usuários, família e sociedade, ou seja, uma visão holística, considerando os múltiplos desdobramentos do fenômeno (econômicos, políticos e sócio-culturais).

Além da carência de conhecimentos sobre a atuação de enfermagem na dependência Química, ainda existe a dificuldade em atuar em comunidades de baixa renda, devido à violência e o medo de sofrer represálias por parte dos traficantes da região, conforme mostra o depoimento:

Abortaram nossa palestra sobre drogas, né, e aí Quando a gente pensou em procurar um líder comunitário pra saber o que ele achava, ele mesmo insistiu Que a gente não tocasse nesse assunto e Que 'não mexesse com Quem tá Quieto, vocês não tem nada a ver com isso, fieuem na de vocês', aí a gente vai por onde? Como é Que a gente vai tratar deste assunto? Adoece o indivíduo, a equipe e a comunidade, a gente fica angustiada em tentar resolver a situação dos pacientes que procuram a gente, encaminhando para outros serviços, vamos escutar, conversar. (E2)

A realidade da violência é polifacetada, se compõe de: exposição freqüente a imagens violentas nos meios de comunicação; testemunhar atos violentos na própria comunidade; sentir-se ameaçado pela violência, fazendo surgir sentimento de impotência; e ainda, o surgimento da crença de Que a violência é o desfecho natural e legítimo para muitos conflitos sociais ${ }^{(17)}$. O profissional de saúde deve analisar a possibilidade de se criarem redes de solidariedade com as organizações governamentais e não governamentais para Que as famílias ao conseguirem identificar seus problemas, aprendam a conhecer-se e ajudar-se mutuamente.

A concepção do cuidado de enfermagem na abordagem ao dependente Químico, segundo o discurso das enfermeiras, tem como base o aconselhamento e o encaminhamento desses usuários a serviços especializados em saúde mental. Dessa forma reproduzse o modelo de institucionalização do dependente Químico, conforme mostram os depoimentos:

Nossa ação é mais no sentido da fala, a gente usa muito este discurso, porQue eu não tenho outra coisa pra oferecer, eu gostaria demais da formação do grupo de adolescentes, [...] $E$ existem os casos Que precisam de internamento, junto com a atenção a saúde mental também, mas em alas diferenciadas, pois na época não tinha CAPS, a gente tinha Que encaminhar Quando ele estava já violento, Quebrando tudo dentro de casa, agredindo a família, criando brigas, Querendo matar os outros, já no último grau. (E I)

Aos que chegam e procuram a gente, nós escutamos, encaminhamos, se vier pra gente pedindo ajuda, a gente encaminha pra outro serviço como o CAPS, a fazenda Nova Esperança, mas assim, Que eu me sinta capacitada pra dar um apoio mesmo, isso me derruba totalmente [...] Quando procuram a gente, nós encaminhamos para outro órgão de ajuda, apoiamos a família, no sentido da escuta, apoio. (E2)

Encaminhamos aos CAPS, onde eles têm lá aQuele apoio social, 
e o Que acho importante se a gente tivesse condições de fazer é trabalhar com o adolescente, Que é uma faixa etária complicada, e muitos destes usuários de drogas estão nesta faixa etária, [...] trabalhando a prevenção primária. (E3)

O dependente Químico tem ficado entre o manicômio e o presídio, ocupando o lugar do louco e do transgressor da lei, ambos excluídos pela sociedade e rotulados ora como doentes e ora como delineuentes ${ }^{(18)}$. A maioria das propostas de tratamento da dependência Química propõe a institucionalização do sujeito, sendo as principais abordagens: médico-farmacológicas, psicossociais, socioculturais e religiosas ${ }^{(19)}$.

Os modelos tradicionais de tratamento da dependência Química baseados na abstinência são os mais difundidos, podendo ser representados pelos Tratamento Moral Pineliano, as Colônias Agrícolas, as Comunidades Terapêuticas e as Fazendas de Recuperação ${ }^{(20)}$. As intervenções em nível hospitalar deveriam se restringir a situações como: síndrome de abstinência grave (alucinações, delirium tremens, heteroagressividade) comorbidades clínicas ou psiQuiátricas severas incontrolável compulsão pelo consumo nocivo de uma ou mais substâncias ou ausência de suporte familiar ou social ${ }^{(21)}$.

A internação em si não é o tratamento, mas apenas uma estratégia de enfrentamento da dependência em situações especiais, objetivando a promoção inicial de abstinência do uso ou a terapia de complicações advindas do consumo abusivo. No restante dos casos, a opção pelo acompanhamento ambulatorial se impõe como a melhor, não excluindo o paciente de seu ambiente e investindo na co-responsabilidade pelo tratamento ${ }^{(21)}$.

Proporcionar tratamento na atenção primária, garantir o acesso a medicamentos, prestar atenção a comunidade, fornecer educação em saúde para a população, envolver comunidades/ famílias/ usuários, formar recursos humanos, monitorizar a saúde mental na comunidade, dar mais apoio à pesquisa e estabelecer programas específicos, são práticas Que devem ser obrigatoriamente contempladas pela política de atenção a usuários drogas, em uma perspectiva ampliada de saúde pública ${ }^{(2 l)}$.

A ESF deve ser vista como uma estratégia de atenção à saúde Que precisa de novos olhares e saberes profissionais, para Que de fato reconheçam as reais necessidades dos usuários. Nessa estratégia o enfermeiro desempenha um papel bastante importante, pois é ele Quem vai acompanhar, supervisionar, promover capacitações, educação continuada com os Agentes Comunitários de Saúde e auxiliares de enfermagem, além de atuar na atividade de cuidar com ênfase na promoção da saúde ${ }^{(22)}$. O campo de atuação para o enfermeiro é grande, ele pode exercer seu papel ao trabalhar a motivação do usuário e o resgate de sua auto-estima em consultas de enfermagem, em visitas domiciliares, em reuniões de grupo de apoio e realizando palestras educativas.

\section{CONSIDERAÇÕES FINAIS}

Os profissionais de enfermagem consideráveis contatos com indivíduos com problemas de alto risco de saúde em conseQüência do uso de substâncias psicoativas, se estes assumem a função de assistência específica a essa população, podem proporcionar cuidados efetivos, prevenindo assim o agravamento dos problemas. O presente trabalho apresenta a importância da intervenção da enfermagem, enQuanto estratégia baseada na recuperação e estabilização do dependente Químico, sendo esta de suma relevância, uma vez Que esse profissional terá mais oportunidades de acesso a esse indivíduo.

Observou-se a atuação do profissional de enfermagem na Atenção Básica de Saúde na abordagem ao dependente Químico em sua prática cotidiana, considerando sua ideologia de trabalho, sentimentos, desejos, temores, interesses, aspirações, seu modo de realizar sua prática e seus comportamentos frente a situações em Que prestou assistência de enfermagem a estes indivíduos.

Constatou-se a carência na formação destes profissionais com relação à temática da dependência Química, restringindo as ações desses profissionais ao encaminhamento desses usuários a serviços mais especializados em saúde mental e, em alguns casos aconselhamento para Que busquem esses serviços. A Universidade como espaço de produção de conhecimentos, não pode ignorar o apelo da sociedade em Que está inserida. Precisa estar atenta para corresponder a estas demandas sociais, preparando os profissionais para intervir nestes cenários. Portanto, enfatiza-se a necessidade dos currículos de enfermagem agregar conteúdos atuais sobre o fenômeno das drogas, preparando os futuros profissionais para o exercício do cuidado nesse campo complexo e prioritário.

Contudo, observamos Que os profissionais entrevistados reconhecem a existência de uma população Que sofre com a dependência Química e necessita de atendimento, porém, relatam Que comumente não exercem atividades específicas voltadas para a esta área. Para tanto, poderia haver a possibilidade de se incluir treinamentos específicos na temática do abuso de drogas. Com essa medida poderia ser possível formar enfermeiros Que teriam idéias e experiências compartilhadas, estratégias bem definidas, conceitos repensados, serviços de apoio indicados e uma linguagem similar abordada. A temática da dependência Química se bem conduzida e trabalhada com as equipes da ESF, poderia contribuir decisivamente para diminuição das internações destes usuários.

\section{REFERÊNCIAS}

1. Organização Mundial de Saúde. Classificação de transtornos mentais e de comportamento da CID- I0. Porto Alegre: Artmed; 2004.

2. Dalgalarrondo P. Psicopatologia e semiologia dos transtornos mentais. Porto Alegre: Artmed; 2000.

3. Centro Brasileiro de Informação sobre Drogas Psicotrópicas CEBRID. II Levantamento domiciliar sobre uso de drogas psicotrópicas no Brasil. São Paulo: CEBRID; 2005.

4. Brasil. Secretaria Nacional Antidrogas. Relatório final do I Fórum Nacional Antidrogas. Brasília: SENAD; 1998.

5. Ximenes Neto FRG, Sampaio IIC. Gerentes do território na Estratégia Saúde da Família: análise e perfil de necessidades de Qualificação. Rev Bras Enferm 2007; 60(6): 687-95.

6. Brasil. Portaria 648 de 28 de março de 2006. Aprova a Política Nacional de Atenção Básica, estabelecendo a revisão de diretrizes e normas para a organização da Atenção Básica para 
o Programa Saúde da Família (PSF) e o Programa Agentes Comunitários de Saúde (PACS). [citado 2009 fev II]. Disponível em: http://dtr2004.saude.gov.br/dab/docs/ legislacao/portaria_648_28_03_2006.pdf.

7. Ministério da Saúde (BR). Secretaria Nacional Antidrogas. Relatório final do I Fórum Nacional Antidrogas. Brasília: Ministério da Saúde; 1998.

8. Luis MAV, Pillon SC. O conhecimento dos alunos de Enfermagem sobre álcool e drogas. Rev Eletrôn Enferm 2003 5(1): 2 I-7. Disponível em: http://www.fen.ufg.br/revista/ revista5_1/pdf/drogas.pdf

9. Minayo M C. O desafio do conhecimento: pesquisa Qualitativa em saúde. $6^{\text {a }}$ ed. Rio de Janeiro: Hucitec-Abrasco; 1999.

10. Instituto Brasileiro de Geografia e Estatística. Censo 2007. Brasília: IBGE; 2007. Disponível em: http://www.ibge.gov.br

11. Fiorin JL. Linguagem e ideologia. $4^{\text {a }}$ ed. São Paulo: Ática; 1995.

12. Ministério da Saúde (BR). Secretaria de Vigilância em Saúde. Secretaria de Atenção à Saúde. Ministério da Saúde. Política na-cional de promoção da saúde. Brasília: Ministério da Saúde; 2006.

13. Carraro T E, Rassol GH, Luis MAV. Nursing formation and the drugs phenomenon in the South of Brazil: nursing students attitudes and beliefs on care. Rev Latino-am Enfermagem 2005; I3(spe): 836-44.
14. Spricigo IS, Alencastre MB. O enfermeiro de unidade básica de saúde e o usuário de drogas: um estudo em Biguaçú-SC. Rev Latino-am Enfermagem 2004; 12(spe): 427-32.

15. Assunção AN. Alcoolismo e ensino de enfermagem: convergências e divergências entre o discurso e a prática [tese]. Pelotas: Universidade Federal de Pelotas; 2000.

16. Barreto AP. Terapia Comunitária passo a passo. $I^{\circ}$ ed. Fortaleza: LCR; 2005

17. Jorge $W$. Periferia e favelização avançam nas grandes cidades da América Latina. Cienc Cult 2005; 57(2): 9-12.

18. Bucher R. Prevenindo contra as drogas e DST/AIDS: cartilha do educador. Brasília: Ministério da Saúde; 1995.

19. Rezende MM. Modelos de análise do uso de drogas e de intervenção terapêutica: algumas considerações. [citado em 2008 maio 25]. Disponível em: http://www.adroga.casadia.org/ tratamento/intervencao.pdf

20. Queiroz IS. Os programas de redução de danos como espaços de exercício da cidadania dos usuários de drogas. Rev Psicol Ciência Prof 200I; 2 I(4): 2-15.

21. Ministério da Saúde (BR). Secretaria Nacional Antidrogas. Conversando sobre cocaína e crack. Brasília: Ministério da Saúde; 2001 .

22. Cortez EA, Tocantins FR. Em busca de uma visão antropológica no Programa de Saúde da Família. Rev Bras Enferm 2006; 59(6): 800-4 